Research Article

\title{
Traffic Condition Aware Customized Beacon Broadcasting (TCA-CBB) Method for Efficient Broadcasting in VANET
}

\author{
S. Sumithra* and R. Vadivel \\ Department of Information Technology Bharathiar University, Coimbatore, 641046, Tamil Nadu, India
}

Received 1 December 2020; Accepted 2 April 2021

\begin{abstract}
In Vehicular Adhoc Network, vehicles need to transmit their original mobility information, in order to maintain the communication reliability for efficient performance of the VANET application. However, some factors such as high broadcasting rate, affect the information accuracy and network reliability. For an effective broadcasting, which maintains network reliability and information accuracy, the broadcasting rate and number of beacons transmitting should be reduced. In this paper, a broadcasting algorithm namely, Traffic Condition Aware Customized Beacon Broadcasting Method (TCA$\mathrm{CBB}$ ) is proposed. TCA-CBB method works based on the traffic condition and adaptively fixes the broadcasting rate according to the current position of the vehicle. Adaptive Extended Kalman Filter (AEKF) is used to predict the current position at broadcasting vehicle side and receiver vehicle side. TCA-CBB is fragmented as (i) Broadcasting Vehicle Segment (BVS) and (ii) Adjacent Vehicle Segment (AVS). Each segment runs a position prediction algorithm called Adaptive Extended Kalman Filter (AEKF), to predict their current position to maintain position accuracy at both the sides. BVS gathers mobility information from Mobility Data Gathering Unit (MDGU) and Traffic Condition Estimation Unit (TCEU) supplies model parameters to the mobility information such as position, speed and direction gathered from MDGU. Self Position Estimation Unit (SPEU) predicts the current position of the Broadcasting Vehicle (BV). Based on the error threshold, the importance of the beacon message is estimated. If the beacon message is important, then it is constructed and scheduled for broadcasting by the Beacon construction and Broadcasting Unit (BCBU). Next Adjacent Vehicle Segment receives the beacon message by Beacon Message Receiving Unit (BMRU). Adjacent Position Estimator Unit (APEU) predicts the current position of the Adjacent Vehicle (AV). The accuracy of the Adjacent Vehicle Position relies on BV's current position. The omitted or lost beacon message is reconstructed using the information shared by the Broadcasting Vehicle. The performance of the proposed method is compared with existing methods such as PPBR, ABR and SAB. The results obtained by the proposed method shows that, TCA-CBB outperformance the existing works.
\end{abstract}

Keywords: Adaptive Kalman Filter, Broadcasting Rate, Mobility Information, Communication Reliability, Position Accuracy, Beacons Message, Error Threshold

\section{.1. Introduction}

Vehicular Ad Hoc Networks provides various kinds of applications for enhancing road safety and entertainment facilities [1]. VANET is constructed of vehicles with OnBoard Unit (OBU), Road Side Unit (RSU) and Centralized Authority (CA). Vehicles can communicate with nearby RSUs (Vehicle-to-Infrastructure) or with each other (Vehicleto-Vehicle) [2]. VANET is specifically designed for providing safety applications and to spread awareness such as cooperative collision warning. Connected vehicles detect the emergencies and spread warning messages to the drivers so that they can take necessary actions such as accident message alters the drivers to take diversion [3]. Modern researchers concentrate on $\mathrm{V} 2 \mathrm{~V}$ communications to provide flexible and reliable safety communication [4]. Every year accidents cause huge fatalities and losses such as injuries, loss of property and loss of time and energy [5]. By enhancing the VANET safety applications, human can obtain potential benefits. In order to enhance the performance of VANET applications, researchers depend on availability and accurate data of recent vehicle trajectories [6]. This information is passed in the form of beacon messages. Due to the dynamic nature of VANET and frequent changes in the environment most of the safety

*E-mail address: sumiphdit@gmail.com ISSN: 1791-2377 @ 2021 School of Science, IHU. All rights reserved. doi:10.25103/jestr.142.04 application require high range of broadcasting messages. in varying vehicle density, high beacon broadcasting is essential to maintain network liveliness and performance of the safety applications [7]. Beacon packets contain the unit block of VANET such as speed, position, direction and acceleration. According to the European and U.S. Standards, it is suggested that the for $1 \mathrm{~km}$ communication range the broadcasting rate to be 10 messages per second $(10 \mathrm{~Hz})$. Regrettably high rate of transmitting beacons definitely create congestion in the communication channel [8]. This may increase when vehicle density is high. In this situation the number of controversy also becomes higher and this results in huge possibility of congestion, high channel usage and low change of accessing the channel. The beacon message containing mobility information will expire soon due to the high velocity of vehicles [9]. Researchers handled this issue by increasing the frequency of the beacon message. But this solution leads to high overhead in the channel. In Urban scenarios, continuous beacon message broadcasting creates congestion in the channel. Other than channel congestion, fading, link breakage and hidden nodes are most troublesome in order to maintain communication reliability. The beacon messages can operate in $5.9 \mathrm{GHz}$ band according to IEEE $802.11 \mathrm{p} /$ WAVE and share a channel part called control channel. The hidden node problem is considered as the serious problem which affects the performance of VANET broadcasting [10]. All these 
issues results in high broadcasting rate which is not suitable for high vehicle density and ephemeral situations and leads to unreliable communication. Final result will be the loss of mobility information. Researchers suggested many broadcasting solutions for adaptive broadcasting depending upon the vehicle density, driving situations and channel characteristics. Researchers aimed to minimize the broadcasting rate so that communication reliability is maintained in a level. But these solutions sacrifice accuracy to maintain reliability [11]. This directly affects the performance of the VANET applications. Therefore the existing broadcasting approaches deals the broadcasting scenario and the mobility prediction scenario in different manner.

The motive of this paper is to concentrate on improving accuracy while maintaining reliability by reducing the broadcasting rate. A new approach called, Traffic Condition Aware Customized Beacon Broadcasting Method (TCA$\mathrm{CBB}$ ) is proposed. As the vehicle status changes frequently, the broadcasting rate may also differ. For Example: for high vehicle density areas, low broadcasting rate is more compatible as well as in low vehicle density areas high broadcasting is suitable. Broadcasting rate is customized based on the vehicle mobility information such as direction and speed. In TCA-CBB, the speed and direction of a vehicle is obtained from the Mobility Data Gathering Unit (MDGU) which employees OIAE-KF. The proposed TCA-CBB method is divided into two major segments. Broadcasting Vehicle Segment (BVS) and Adjacent Vehicle Segment (AVS). First, Traffic Condition Estimation Unit (TCEU), Self Position Estimator Unit (SPEU) and Beacon Construction and Broadcasting Unit (BCBU) construct Broadcasting Vehicle Segment. TSEU obtains the mobility characters from the MDGU and build the mobility model parameters using time series Autoregressive Yule Walker method AR(p). SPEU predicts the sender vehicle's current position by using Adaptive Extended Kalman Filter (AEKF). This unit maintains the accuracy of the sender position estimation by fixing a threshold. BCBU is responsible for deciding whether to broadcast the mobility message or not. The beacon message is constructed only when the prediction accuracy is greater than the threshold value. BCBU takes care of the beacon message construction and the beacon broadcasting. Such that broadcasting rate is reduced according to the vehicle behavior and the traffic condition by transmitting the beacon messages as per the prediction accuracy threshold. Second, Adjacent Vehicle Segment (AVS) is build by Beacon Message Receiving Unit (BMRU) and Adjacent Position Estimator Unit (APEU). BMRU is responsible for receiving the incoming beacons which is constructed using predicted sender position, mobility model parameters and the threshold value. APEU estimates the position of the adjacent vehicle using AEKF. This prediction helps to predict the omitted or lost beacon messages.

In this paper is organized as following sections. Section I explains the introduction part of the research work. Section II talks about the literature survey conducted based on the research work. Section III demonstrates the proposed work in detail. Section IV illustrates the performance evaluation, simulation and the results obtained by the proposed work. Final section concludes the research work.

\section{Related Works}

Modern research analysis proves that ordinary fixed broadcasting rate is not much appropriate for extremely dynamic traffic situations such as Vehicular Network. Fixed broadcasting rate affects both VANET applications and their performance such as communication cost. This results in inaccurate measurements. Previous findings shows there are many adaptive broadcasting approaches, which can be classified based on adapting the broadcasting rate, adapting the transmission power, and hybrid adaptation. The first approach adaptive broadcasting rate is aimed to take the broadcasting rate suitable for specific situations like high channel occupying time, high vehicle density and high message drop. This approach mainly aims to reduce the broadcasting beacons and enhance the communication reliability. The second approach of adapting transmission power provides high communication reliability by reducing the number of communicating vehicles. This is done by lessening the communication range with fixed broadcasting rate. Chen and Chang [12] suggested that transmission power control has an disagreed impact on communication trustworthiness. This approach splits the congestion part into several broadcasting regions. Due to this divided regions, the vehicles are unable to detect each other signals. As the result, the hidden terminals may increase and congestion is created. Medium access control methods bring homogeneous distribution while Transmission power control approach brings heterogeneous distribution. The third approach is the combination of broadcast rate adaptation and transmission power adaptation to enhance the efficiency of communication channel utilization. Even though, this approach also has some demerits such as not considering information accuracy. Yongtae Park [13] suggested an application level messaging frequency estimation scheme called Frequency Adjustment with Random Epochs (FARE). This approach significantly improves the Basic Safety Message (BSM) using less bandwidth than IEEE 802.11p.

To predict the omitted information from the previously received beacon, each object vehicle uses a position deduction model called Neighbor-Estimator for each neighbor. Here the threshold value is fixed to 0.3 meters lateral and 0.5 meters for longitudinal errors. Neighbor estimator model works based on dead reckoning by kinematic equations from the vehicle. This model is aimed to reduce the rate of broadcasting while maintaining the accuracy. But some assumptions like ideal communications are unrealistic for VANET which produces inefficient results. S. Rezaei et al. [14] proposed an adaptive communication scheme for Cooperative Active Safety System (CASS). This approach uses a vehicle to frequently broadcast safety related information for every $100 \mathrm{~ms}$. The simulations shows CASS could perform better for every $500 \mathrm{~ms}$. Nevertheless the linear vehicle movement and the ideal communication are assumed. This produces unrealistic results. Another estimator called Remote-Estimator, which predicts present position from previous position estimated using Self-Estimator. Schmidt et al. proposed Situation Adaptive Beaconing (SAB) approach, which depends on vehicle's status and the road traffic situation [15]. In addition, SAB is categorized into two rate adaptation schemes depending on vehicle's movement and the neighbor vehicle movement. However, the minimization of broadcasting beacon rate is not concentrated here. Ghafoor et al. [16] proposed an Intelligent Adaptive Beaconing Rate (ABR) approach depending upon fuzzy logic to maintain the frequency of beacon broadcasting by considering traffic characteristics. ABR works based on same direction the vehicles are travelling. The status of the vehicles is the input 
of the fuzzy decision making system. This induces to adjust the beaconing rate according to the traffic characteristics. The ABR system showed improvement in communication reliability in dynamic situations for certain point. But in this scheme the impact of accuracy of beacon information and the communication reliability is not considered. Moreover, the ABR scheme cannot cope up with emergency situations such as declaration or lane changing behavior. Variables such as channel busy time, vehicle density and the rate of collision are extremely dynamic even in minimum time intervals. Zemouri at al. [17] proposed an approach to handle the congestion problem in channel. Authors introduced a novel vehicle centric short-term density prediction scheme. This scheme estimates the density of the vehicles inside the communication range of a given vehicle. The vehicles should adapt their current state of network as parameters within the next time window. This is a hybrid scheme of adapting both power transmission and broadcasting rate. This approach enhances the network performance but the accuracy achieved is not analyzed. Liu and Wang [18] proposed a Position Prediction Based Beacon Rate (PPBR) approach based on position prediction to decrease bandwidth consumption by reducing beacon frequency. In this approach instead of using periodic beacon broadcasting, the vehicles track their neighbors by predicted position. This method induces the beacon broadcasting only when the prediction error exceeds the predefined tolerance. A switching approach was designed based on maneuvering detection to swap between the motion types. However this approach doesn't come up with highly dynamic situations. Yin, et al. [19] proposed an analytical model for MAC and Application level performance evaluation in dedicated short-range communications (DSRC) for periodic beacon message dissemination.

On summing up, the performance of the broadcasting approaches in VANET clearly depends upon the accuracy of the prediction measurements in both sender and adjacent vehicles. But due to the ephemeral nature of VANET, vehicle mobility characteristics such as speed, direction and position may change rapidly. Vehicle may enter in and go out another vehicle's communication range within a second. This creates frequent link broken communications which leads to asymmetrical arrival of beacons from the sender vehicles. Due to this situation the information from the broadcasted beacon is highly unreliable. The existing broadcasting approaches concentrated on reliability of the information skipping the accuracy [20]. In this paper, the accuracy of the mobility information is focused. By using this, at highly dynamic traffic conditions, broadcasting rate is customized based on the threshold. Therefore, the enhanced accuracy of sender prediction and the adjacent prediction reduces number of broadcasting messages and improving the communication reliability automatically.

\section{Traffic Condition Aware Customized Beacon Broadcasting Method (TCA-CBB)}

The aim of the proposed TCA-CBB method is to minimize unwanted transmissions in VANET while maintaining security information accuracy for achieving reliability and accuracy at the same time [21]. In dynamic traffic situations such as high density and low density environments, high and fixed broadcasting rate is not suitable. Based on the traffic condition, each vehicle is allowed to choose their broadcasting rate. Therefore broadcasting decision is an individual decision. In VANET the mobility information of the vehicle is associated with time, so current mobility information is calculated from previously received mobility information. In this paper the vehicle which broadcasts the mobility information is considered as Broadcasting Vehicle (BV) whereas the vehicle which receives the mobility information is called Adjacent Vehicle (AV). Both the BV and AV segments will be placed in a single vehicle. Because a vehicle could broadcast a message as well as receive a mobility message. First Segment is the Mobility Data Gathering Unit (MDGU), which is responsible for predicting the current position of the vehicle. The broadcasting vehicle's position, velocity and direction are obtained here by using Optimal Innovation Based Adaptive Estimation Kalman Filter (OIAE-KF). Broadcasting Vehicle Segment (BVS) is assembled based on Mobility Data Gathering Unit (MDGU), Traffic Condition Estimation Unit (TCEU), Self Position Estimator Unit (SPEU) and Beacon Construction and Broadcasting Unit (BCBU). TCEU is used for building mobility parameters using the mobility information such as current position, velocity and the direction from OIAE-KF in MDGU. Traffic Condition based prediction model parameters are short term patterns trained from the previously estimated mobility models. SPEU uses Adaptive Extended Kalman Filter (AEKF) for to estimate the broadcasting vehicle's next mobility state using the short term model parameters from TCEU. By comparing the error produced by the self position estimator and the error tolerance threshold, the BV decides whether to broadcast the mobility information or not. Beacon Construction and Broadcasting Unit (BCBU) constructs the beacon message if $\mathrm{BV}$ decides to broadcast the message. In the beacon message packet, BV attaches the direction and speed model parameters along with the noise covariance of the AEKF algorithm. Adjacent Vehicle Segment (AVS) is responsible for receiving the beacon messages and predicts the omitted or lost messages by the mobility model parameters received from beacon packets.

\subsection{Broadcasting Vehicle Segment (BVS)}

As shown in figure 1, the Broadcasting Vehicle Segment (BVS) holds four main units such as Mobility Data Gathering Unit (MDGU), Traffic Condition Estimation Unit (TCEU), Self Position Estimator Unit (SPEU) and Beacon Construction and Broadcasting Unit (BCBU). Optimal Innovation based Adaptive Estimation Kalman Filter (OIAE$\mathrm{KF}$ ) is built in MDGU unit, which send the information like vehicle's current position, velocity and direction. This information is obtained in an accurate and timely manner. OIAE-KF integrates the kinematic measurements and the positioning measurements together to produce accurate current positioning information.

\subsubsection{Traffic Condition Estimation Unit (TCEU)}

Traffic Condition Estimation Unit is indented to obtain the short-term model parameters such as speed and direction. The speed and direction of a vehicle is converted to an autoregressive model with $p$ order. $A R(p)$ model is a representation of linear regression process of previous values and the current values based on the time series. (i.e.) the new output values linearly depend upon the past values. There are numerous techniques to estimate AR (p) coefficients. In this research article Yule-Walker method is used for modeling speed and direction parameters. The value of $p$ is the order of autoregressive model, which represents the current value in the time series [22]. The value $\mathrm{p}$ is a zero mean stationary process. The following equation shows the general autoregressive process. 
$X_{t}=\phi_{0}+\phi_{1} X_{t-1}$

A more sufficient and generalized model is $\mathrm{AR}(\mathrm{p})$ model which is shown in the following equation.

$X(t)=\phi_{1} X(t-1)+\phi_{2} X(t-2)+\cdots$

$+\phi_{p} X(t-p)+a_{t}$

As mentioned previously, $p$ is the order. The value $a_{t}$ is the white noise with constant finite variance $\sigma_{a}^{2}$ and zero mean. The illustration of the AR (p) model contains the form of linear regression model when $X_{t}$ is set as the dependent variable. The lagged values $X(t-1), X(t-2), \ldots . X(t-$ $p)$ are then set as the advisory variable. Nevertheless, there are some variations between the two models. In this paper, the past $p$ values $X(t-1)(\mathrm{i}=1, \ldots, \mathrm{p})$ decides the conditional expectation of $X_{t}$ obtained from the previous data. The final $\mathrm{AR}(\mathrm{p})$ equation is given as follows.

$$
\phi=C^{(-1)} b
$$

Where $C$ is the auto covariance matrix, $\mathrm{b}$ is the autocorrelation vector and $\varphi$ is the vector which holds the model parameters which are unknown. It can be shown that:

$$
\sigma_{a}^{2}=\sum_{t=0}^{p} \phi_{t} \rho(t)
$$

By solving the above equation, the model parameters are obtained for prediction. The current mobility information of the broadcasting vehicle is placed in a measurement vector $y_{k}^{B V}$ at time epoch $\mathrm{k}$. The TCEU, outputs the short-term model parameters by taking the speed and direction of the broadcasting vehicle as the input. Model parameters are denoted as $\phi_{k}$ and the uncertainties obtained from the model parameters are denoted as $\sigma^{2}$.

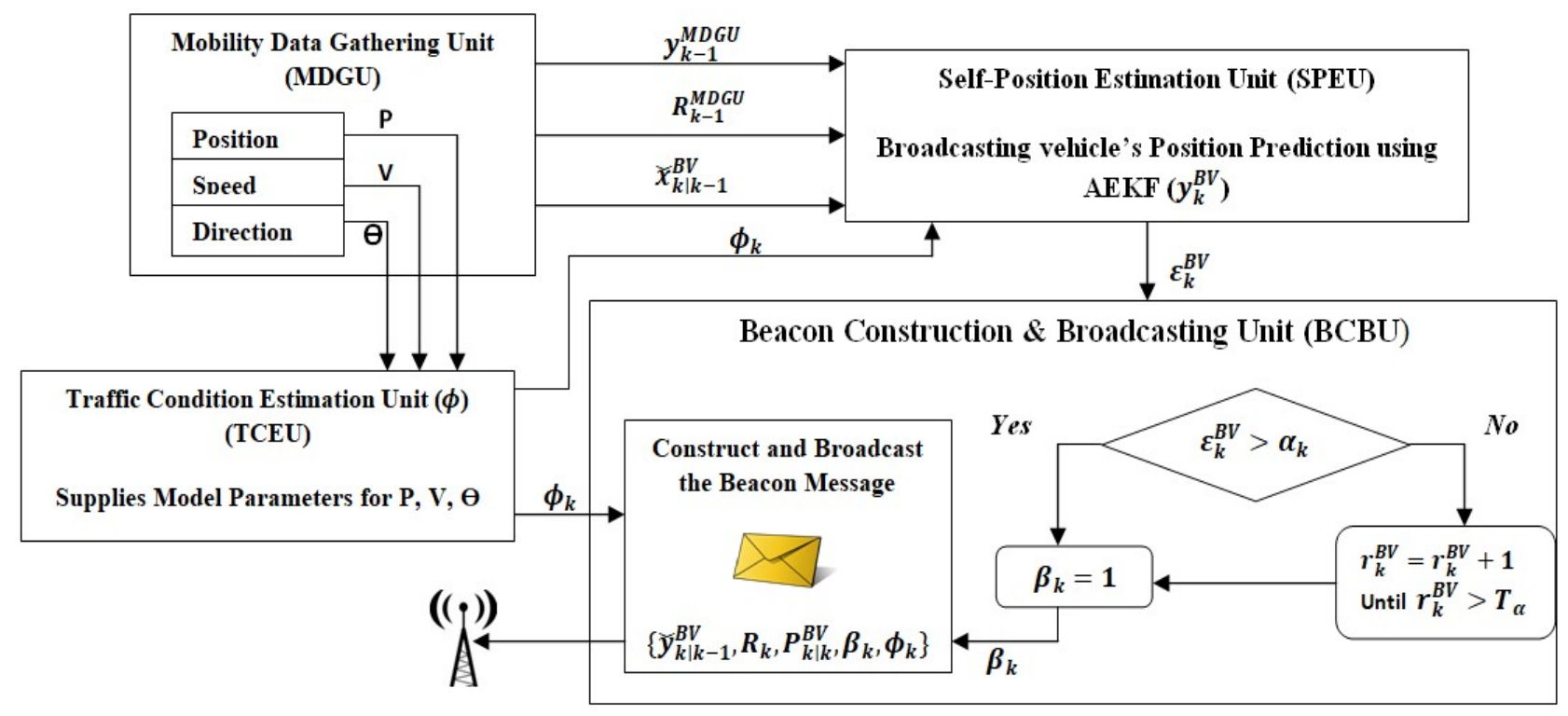

Fig. 1. Broadcasting Vehicle Segment (BVS)

\subsubsection{Self Position Estimator Unit (SPEU)}

Next Unit is the Self Position Estimator Unit (SPEU) takes the coefficients from TCEU as an input along with two vectors such as measurement vector $y_{k}^{B V}$ of the broadcasting vehicle at the time epoch $\mathrm{k}-1$ and the second vector is the measurement noise covariance $R_{k-1}$. The output of the SPEU is the upcoming position measurement prediction $y_{(k \mid k-1)}^{B V}$. The broadcasting vehicle runs a unit called Self Position Estimator Unit (SPEU), which predicts its future position. This future position is send to the adjacent vehicle for predicting their position so that the adjacent vehicle could maintain accurate position. SPEU uses Adaptive Extended Kalman Filter (AEKF) to predict the vehicle's current mobility information. OIAE-KF supplies the speed and direction measurements to the AEKF obtained at a time epoch.

To drive the Self Position Estimation Unit, have to consider the following dynamics:

Priori Mobility State $=\breve{x}_{(k \mid k-1)}^{B V}$

Previous State $=\breve{x}_{k-1 \mid k-1}^{B V}$
Unknown model parameters vector at time epoch $=\phi_{k}$ Some parameters need to be initialized before starting the AEKF process. These parameters are residing in MDGU. The model for prediction is $f_{k}\left(\breve{x}_{(k-1 \mid k-1,)}^{B V} \phi_{k}\right)$, the measurement noise covariance $R_{k}$ of measurement noise vector $v_{k-1}$ and the process noise covariance $Q_{k-1}$ of process noise vector $w_{k-1}$. The following equation illustrates the AEKF measurement process.

$\breve{x}_{k \mid k-1}^{B V}=f_{k}\left(\breve{x}_{k-1 \mid k-1,}^{B V} \phi_{k}\right)+w_{k-1} \breve{x}_{k \mid k-1}^{B V}=$

$f_{k}\left(\breve{x}_{k-1 \mid k-1,}^{B V} \phi_{k}\right)+w_{k-1}$

$\breve{y}_{k \mid k-1}^{B V}=H_{k}\left(\breve{x}_{k \mid k-1}^{B V}+v_{k-1}\right)$

\subsubsection{Prediction Process}

First, acquire the state $\bar{x}_{k-1 \mid k-1}^{B V}$ at k-1 time epoch. Get the linear form of the prediction model in the region of vector components of the matrix $F_{k}$. The mapping matrices $f_{k}(x)$ and $h_{k}(x)$ both are non-linear from equation 7 and equation 8.

$F_{k}=\frac{\partial f_{k}}{\partial x}\left(\breve{x}_{k-1 \mid k-1}^{B V}\right)$ 


$$
H_{k}=\frac{\partial f_{k}}{\partial x}\left(\breve{x}_{k \mid k-1}^{B V}\right)
$$
Filte

Apply the and the $H_{k}$ to the Adaptive Extended Kalman

$$
\begin{aligned}
& \mathrm{r} \breve{x}_{k \mid k-1}^{B V} \text { and } \breve{P}_{k \mid k-1}^{B V} \\
& \breve{x}_{(k \mid k-1)}^{B V}=F_{k} \breve{x}_{(k-1 \mid k-1)}^{B V} \\
& \breve{P}_{(k \mid k-1)}^{B V}=F_{k} \breve{P}_{(k \mid k-1)}^{B V} F_{k}^{T}+Q_{k}
\end{aligned}
$$

Where $Q_{k}$ is the uncertainty or measurement noise covariance, which is equal to $\sigma^{2}$.

\subsubsection{Measurement Process}

Current mobility state of the broadcasting vehicle $\breve{y}_{k \mid k-1}^{B V}$ is calculated by SPEU. Now, to obtain the error or residual mobility measurement, the actual mobility measurement $y_{k}^{B V}$ is gathered from MDGU. By subtracting the SPEU mobility measurement and the actual mobility measurement, the innovation error $\varepsilon_{k}^{B V}$ is obtained.

$\varepsilon_{k}^{B V}=y_{k}^{B V}-\breve{y}_{k \mid k-1}^{B V}$

The innovation error is used for obtaining the error tolerance threshold $\alpha_{k}$ in the Beacon Construction and Broadcasting Unit (BCBU). This importance is stored in $\beta$. When the innovation error exceeds the threshold value, the mobility information is considered as important (i.e) $\left(\beta_{k}=1\right.$ ). If $\beta_{k}=0$ then the mobility message is not important.

\subsubsection{Correction Process}

Correction process is used to predict the mobility information $y_{k}^{D R}$ using Dead Reckoning method using vehicle's constant acceleration kinematic model (vehicle sensors measurements). This is helpful when the $\varepsilon_{k}^{B V}$ is lower than $\alpha_{k}$ for longer time. (i.e) $\beta_{k}=1$. Once the $\beta_{k}$ is set to 1 , (i.e) $\varepsilon_{k}^{B V}>\alpha_{k}$, the threshold value is updated and trained for a new mobility model. Innovation sequence $e_{k}$ is calculated. Kalman Gain is calculated and the corrected phase is applied to the AEKF. At the end of the AEKF process, the final predicted state $\breve{x}_{(k \mid k)}^{B V}$ is estimated at time epoch $k . \breve{x}_{k \mid k}^{B V}$ is used for estimating the future mobility state $\breve{x}_{k+1 \mid k}^{B V}$ at the $k+1$ time epoch. Uncertainties of the final prediction $\breve{P}_{k \mid k}^{B V}$ to be send along with the beacon message is also estimated.

$e_{k}=\breve{y}_{k \mid k-1}^{B V}-y_{k}^{D R}$

$K_{k}=\breve{P}_{k \mid k-1}^{B V} H_{k}^{T}\left(H_{k} \breve{P}_{k \mid k-1}^{B V} H_{k}^{T}+R_{k}\right)^{-1}$

$\breve{x}_{k \mid k}^{B V}=\breve{x}_{k \mid k-1}^{B V}+\beta_{k} K_{k} \varepsilon_{k}^{B V}$

$\breve{P}_{k \mid k}^{B V}=\left(I-\beta_{k} K_{k}\right) \breve{P}_{k \mid k-1}^{B V}$

\subsubsection{Beacon Construction and Broadcasting Unit (BCBU)}

In the Beacon Construction and Broadcasting Unit (BCBU), broadcasting decision is based on the error obtained from the SPEU which is denoted as $\varepsilon_{k}$. By subtracting the $y_{k \mid k-1}^{\mathrm{BV}}$ by $y_{k}^{\mathrm{BV}}$, error of SPEU $\varepsilon_{k}$ is obtained. Based on the error, the broadcasting vehicle decides whether to schedule the beacon message or not. When $\varepsilon_{k}^{\mathrm{BV}}$ exceeds the threshold value $\alpha_{k}$, the mobility message is considered as important and scheduled for broadcasting. If $\varepsilon_{k}^{\mathrm{BV}}$ is less than the threshold value $\alpha_{k}$ the mobility message is not important. If $\varepsilon_{k}^{\mathrm{BV}}$ is less than $\alpha_{k}$ for a long while, it should reach the maximum updating threshold value $T_{\alpha}$. This happens when vehicle does not change its situation. The sampling interval time is counted by a variable $r_{k}^{\mathrm{BV}}$, to reach $T_{\alpha}$. Hence, a mobility message is considered as important and ready for broadcasting is any one of the threshold condition is reached. i.e $\left(\varepsilon_{k}^{\mathrm{BV}}>\alpha_{k} \| r_{k}^{B V}>\mathrm{T}_{\alpha}\right)$ . At the end of the iteration, the mobility message is set either important or not important. This importance is stored in $\beta$. (i.e) $(\beta=1)$. The threshold value is updated frequently according to the information accuracy obtained from the current communication state in order to avoid unwanted communication during varying traffic conditions. Threshold value is fully depending upon the accuracy achieved from the Adjacent Position estimation Unit (APU) after predicting the adjacent vehicle position. When all the conditions are satisfied (i.e $\beta$ is set to 1 ), the beacon message is constructed and ready to broadcast. Figure 2 shows the algorithm for Broadcasting Vehicle Segment.

$$
\begin{aligned}
& \alpha_{k}=\left\{\begin{array}{l}
1, \varepsilon_{k}^{B V} \geq \beta_{k} \\
0, \varepsilon_{k}^{B V}<\beta_{k}
\end{array}\right. \\
& y_{k}^{B V}=\left\{y_{k}^{M D G U}, \phi_{k}, R_{k}, P_{k \mid k}^{B V}, \beta_{k}\right.
\end{aligned}
$$

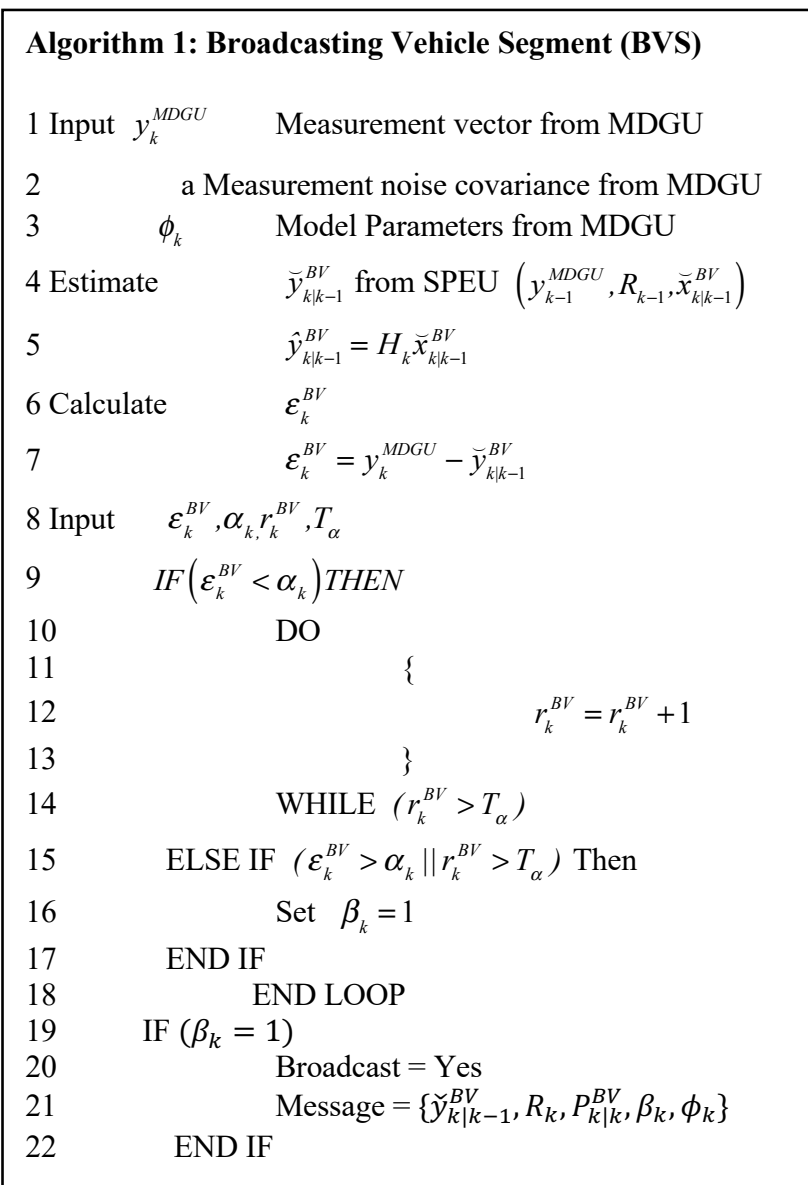

Fig. 2. Algorithm for Broadcasting Vehicle Segment (BVS)

\subsection{Adjacent Vehicle Segment (AVS)}

This segment works when an adjacent vehicle receives a beacon messages from a broadcasting vehicle. Broadcasting vehicles are tending to maintain the status of the adjacent vehicles that pass through the communication region of the 
broadcasting vehicle. Vehicles have same service time due to the synchronization between them within the vehicle communication region. Synchronization is done based on the Local Dynamic Map (LDM). LDM share the mobility related information, which are, gathered with various frequency intervals from several vehicles. LDM provides reliable and real time dynamic view of the traffic situation for the VANET applications [23]. As all vehicles are connected with GPS which provides a real time service so synchronization is possible and valid assumption. It is assumed that the common position information of the vehicles is stored in LDM. Adjacent Vehicle Segment contains two parts. Beacon Message Receiving Unit (BMRU) and Adjacent Position Estimation Unit (APEU). Figure 3 shows the diagrammatic representation for Adjacent Vehicle Segment.

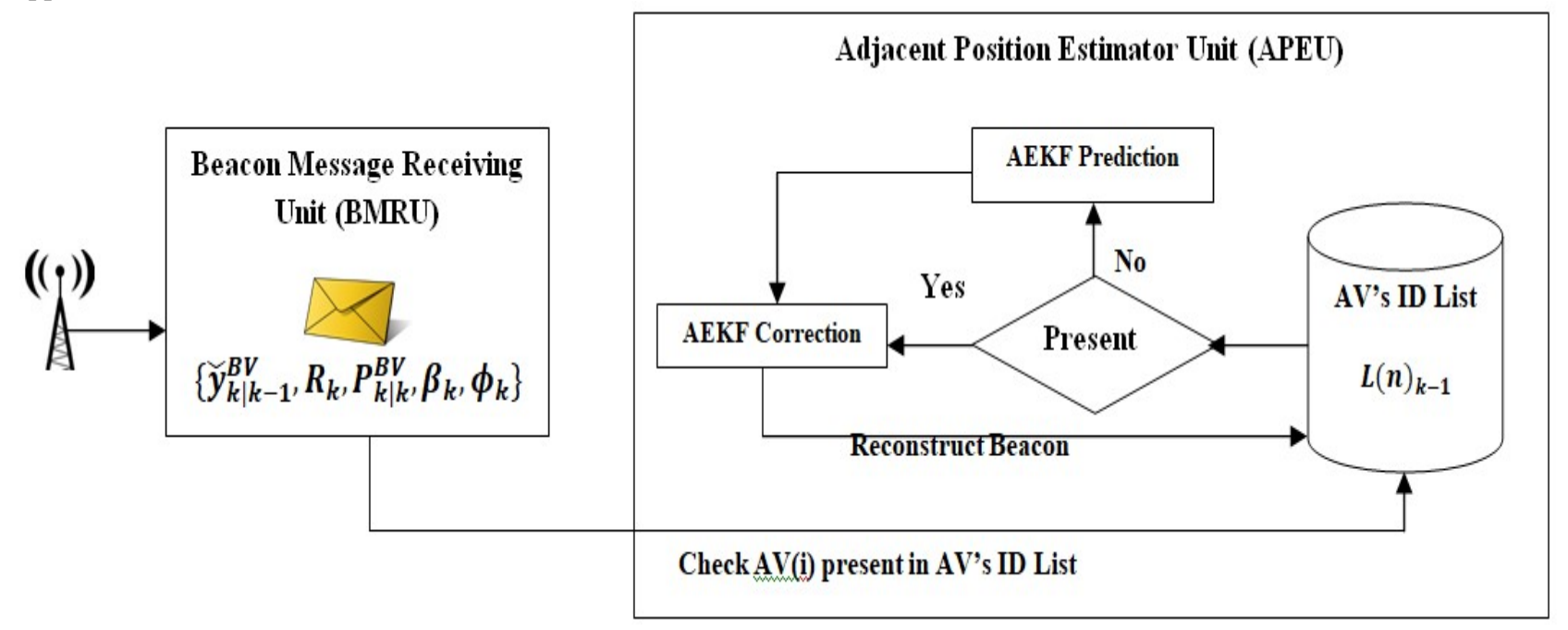

Fig. 3. Adjacent Vehicle Segment

\subsubsection{Beacon Message Receiving Unit (BMRU)}

Beacon Message Receiving Unit (BMRU) receives the beacon message from the broadcasting vehicle. The channel access time is subdivided into $100 \mathrm{~ms}$. This is called Synchronize Interval (SI) [24]. SI is divided as Control Channel Interval (CCHI) \& Service Channel Interval (SCHI). Each contains $50 \mathrm{~ms}$. All the vehicles in VANET are synchronized using GPS transceiver in build in the On Board Unit (OBU) of the vehicles. Every vehicle in VANET broadcast or receives safety related information through CCHI. Non-safety or service related information is transmitted through SCHI. Let $L(n)_{k-1}$ be the vector, which holds the list of $n$ number of adjacent vehicle IDs at time epoch $\mathrm{k}-1 . \lambda_{k-1}^{A V}$ is the vector that holds the messages received at time epoch k-1. $\breve{x}_{k}^{A V}$ is the vector which holds the total beacon messages received at $\mathrm{k}$ epochs time. There are two situations for each beacon message $\lambda_{k-1}^{A V}$. One is the broadcasting vehicle is previously listed in the AV's adjacent vehicle list $L(n)_{k-1}$ and second one is not listed. If the Broadcasting Vehicle (BV) is already listed in the neighbor list of AV, $L(n)_{k-1}$, the beacon arrival status $a_{k}^{i}$ is set to 1 at k epoch time $a_{k}^{i}=1$. The beacon messages which are not listed in the AV's neighbor list $L(n)_{k-1}$, then the ID of the BV which broadcasted the beacon message is enclosed with the neighbor list of AV. In some cases, at the end of CCHI $50 \mathrm{~ms}$, the $\mathrm{BV}$ is present in the neighbor list $L(n)_{k-1}$ but beacon message is still not received. In that situation, the arrival status is set to zero $a_{k}^{i}=0$. A copy of AEKF prediction process is applied in the adjacent vehicle for predicting more accurate adjacent vehicle position.

\subsubsection{Adjacent Position Estimator Unit (APEU)}

At the adjacent vehicle side, a copy of the SPEU is processed as Adjacent Position Estimator Unit (APEU). APEU is fully dependent on the reliability of the SPEU. The reliability is assured in the SPEU based on the threshold limit. Each received beacon message $B_{k}^{i}$ assigned as 1 which is a binary value. This is to notify that the beacon message arrival status is successful from vehicle $i$ in in the adjacent vehicle state vector $A V_{k-1}$. After executing the copy of the AEKF in APEU, the following measurement is obtained.

$y_{k}^{A V(i)}=H_{k} \breve{x}_{k \mid k-1}^{A V(i)} B_{k}^{i}$

The innovation sequence is obtained by subtracting the measured value and the actual value as follows

$\breve{e}_{k}=y_{k}^{A V(i)}-\breve{y}_{k \mid k-1}^{A V(i)}$

By using the uncertainties such as measurement noise covariance $R_{k}$ and final uncertainty $P_{k \mid k}^{B V}$ enclosed along with the beacons, Kalman gain is calculated. If the beacon message is lost or does not arrive, the measurement noise covariance $R_{k}=\sigma^{2} I$. The covariance $\sigma^{2}$ grows with time. If a beacon is lost, the broadcasting time interval will becomes longer. In such case, $R_{k}$ reaches higher value. Therefore, the estimation made by AEKF is stable and moderate. When beacon is lost the adjacent vehicle again estimates the $R_{k}$. The final state estimation $\breve{x}_{k \mid k}^{A V(i)}$ is the prediction result of the APEU based on the beacon message at $k$ time epoch with recreating the beacon message $y_{k}^{A V(i)}$ when it is lost. Figure 4 shows the algorithm for Adjacent Vehicle Segment. 


\begin{tabular}{|c|c|c|}
\hline \multicolumn{3}{|c|}{ Algorithm 2: Adjacent Vehicle Segment (AVS) } \\
\hline \multicolumn{3}{|c|}{1 Input $L(n)_{k-1}$ Vehicle ID at k-1 time epoch } \\
\hline 2 & $x_{(k-1)}^{A V}$ & Status vector of $\mathrm{n}$ AVs at k-1 time epoch \\
\hline 3 & $\lambda_{(k-1)}^{A V} \quad \mathrm{~V}$ & ector for receiving message at k-1 \\
\hline 4 & $\breve{x}_{k}^{A V}$ & Tector of beacon received in current time \\
\hline \multicolumn{3}{|l|}{ k. } \\
\hline 5 & $A V(i) \quad \mathrm{Ad}$ & jjacent Vehicle ID \\
\hline \multicolumn{3}{|c|}{6 FOR EACH $A V(i)$ check } \\
\hline 7 & \multicolumn{2}{|c|}{ IF $(A V(i))$ present in $L(n)_{k-1}$ Then } \\
\hline \multicolumn{3}{|c|}{$\lambda_{(k-1)}^{A V}=1$} \\
\hline 9 & \multicolumn{2}{|r|}{$K_{k}=\breve{P}_{k \mid k}^{A V(i)} H_{k}^{T}\left(H_{k} \breve{P}_{k \mid k}^{+} H_{k}^{T}+\breve{R}_{k}^{A V(i)}\right)^{-1}$} \\
\hline 10 & \multicolumn{2}{|r|}{$\breve{x}_{k \mid k}^{A V(i)}=y_{k}^{B V(i)} H_{k}^{-1}$ from BV } \\
\hline 11 & \multicolumn{2}{|r|}{$\breve{P}_{k \mid k}^{A V(i)}=P_{k \mid k}^{B V(i)}$ from BV } \\
\hline 12 & \multicolumn{2}{|r|}{$R_{k}^{A V(i)}=R_{k}^{B V(i)}$ from $\mathrm{BV}$} \\
\hline 13 & \multicolumn{2}{|l|}{ ELSE } \\
\hline 14 & \multicolumn{2}{|r|}{$\lambda_{(k-1)}^{A V(i)}=0$} \\
\hline 15 & \multicolumn{2}{|r|}{$L(n)_{k-1}=L(n)_{k-1}+A V(i)$} \\
\hline 16 & \multicolumn{2}{|r|}{$K_{k}=\breve{P}_{k \mid k-1}^{A V(i)} H_{k}^{T}\left(H_{k} \breve{P}_{k \mid k-1}^{A V(i)} H_{k}^{T}+\widehat{R}_{k}^{A V(i)}\right)^{-1}$} \\
\hline 17 & \multicolumn{2}{|r|}{$\breve{x}_{k \mid k}^{A V(i)}=\breve{x}_{k \mid k-1}^{A V(i)}+K_{k} \breve{e}_{k}$} \\
\hline 18 & \multicolumn{2}{|r|}{$\breve{P}_{k \mid k-1}^{A V(i)}=\left(I-K_{k} H\right) \breve{P}_{k \mid k-1}^{-}$} \\
\hline \multicolumn{2}{|c|}{$\begin{array}{l}19 \quad \text { END IF } \\
20 \text { END LOOP }\end{array}$} & \\
\hline
\end{tabular}

Fig. 4. Algorithm for Adjacent Vehicle Segment (AVS)

\section{Performance Evaluation}

This section describes the simulation setup and the simulation process, along with the results obtained from the simulation. The simulation starts with two phases, such as traffic simulation and network simulation. Simulation for Urban Mobility (SUMO) generates road traffic, and Network Simulator 2 (NS2) runs the network simulation. MATLAB is used for computation. Next Generation Trajectory Data set is used to simulate the proposed method [25]. Simulation is used to evaluate the effectiveness of the proposed method. Next Generation Simulation (NGSIM) data set is the collection of the trajectories collected from the real world vehicles. This data set is used to build the realistic mobility modes to under the driver behavior. The effectiveness of the proposed TCACBB method has been simulated based on fixed broadcasting intervals and varying driving behavior [26]. The effective performance of the proposed method depends upon the robustness of the prediction process of the Broadcasting Vehicle (BV) and the Adjacent Vehicle (AV). This factor helps to achieve adequate accuracy under varying driving behavior and different situations. Two types of broadcasting rates such as fixed and customized broadcasting rates are conducted for evaluating the proposed method. Three existing broadcasting methods such as Situation Adaptive Beaconing (SAB) [15], Adaptive Beaconing Rate (ABR) [16] and Position Prediction Beacon Rate (PPBR) [18] are used for comparison. These existing works are the most related and recent works done previously for VANET broadcasting. To setup the VANET wireless channel, 2-ray ground reflection model is used. Every vehicle in the VANET environment has the communication range of 250 meters. This implements the physical layer for transmission. As seen before, SUMO is used for establishing the traffic model. An .osm file of Coimbatore city is downloaded from the Open Street Map (OSM) location which is shown in figure 5. The maximum speed of a vehicle is $20 \mathrm{~m} / \mathrm{s}$. The simulation area is fixed to $1000 \times 1000$ meters (Coimbatore City area). Totally 200 vehicles are used for simulation. For MAC layer protocol stack, IEEE Standard 802.11 Distributed Coordination Function (DCF) is used. Channel Bandwidth is $3 \mathrm{Mbps}$. The interface queue between MAC layer and Data Link Layer (DLL) is used for storing packets waiting for channel access with maximum of 25 packets. Constant Bit Rate with a value of $35 \mathrm{Kbps}$ is the traffic source of simulation [27]. This traffic source is the UDP packets generation type. Totally 10 vehicles are chosen to transmit the data packets. The transmitting packet size is 1000 bytes. The simulation time is set to 500 seconds. To remove the barriers that affect the simulation results, the settling time is set to 25 seconds. Figure 6 shows the network file running in SUMO. Table 1 illustrates the simulation parameters used.

Table 1. Simulation Settings

\begin{tabular}{l|l}
\hline Simulation Parameters & Values \\
\hline Wireless Channel & 2-ray channel \\
Traffic Simulator & SUMO \\
Network Simulator & NS 2.34 \\
Map Model & OSM (Coimbatore City) \\
Max Speed & $20 \mathrm{~m} / \mathrm{s}$ \\
Simulation Area & $1000 \times 1000$ \\
Number of Vehicles & 200 Vehicles \\
Transport Protocol TCP & UDP \\
Routing Protocol DSR & DSDV \\
Communication Range & $250 \mathrm{~m}$ \\
MAC Layer & IEEE 802.11 \\
Channel Bandwidth & $3 \mathrm{Mbps}$ \\
Simulation time & $500 \mathrm{~seconds}$ \\
\hline
\end{tabular}

\subsection{Simulation setup}

In this paper, the noises in NGSIM measurements such as speed and acceleration are smoothened using Exponentially Weighted Moving Average (EWMA), which places a weight and importance to the most recent measurements. The position displacement of the vehicle from $\mathrm{x}$-axis to $\mathrm{y}$-axis gives the heading angle and the speed of the vehicle gives the acceleration. Four different types of clusters such as lane changing, car flowing, free flowing and random flowing are formed based on the NGSIM dataset. K-means clustering method is used for clustering. The advantage of this clustering is including all types of traffic behaviors in the simulation and committing all driver behaviors in the proposed work. The proposed scheme aims to achieve low broadcasting and fetch high accuracy. Each cluster contains 50 samples for evaluation and totally 200 data samples are used. Samples are selected in random based on the distance of the cluster head. Beacon message arrival rate is the key factor for communication reliability. Few factors influence the beacon arrival rate such as beacon size, vehicle density, environmental conditions and problems in MAC layer [28]. The beacon arrival rate and loss rate depends upon the variable scenarios. In this paper, the beacon message loss is considered based on different vehicle densities. Table 2 represents different vehicle density scenario with $100 \mathrm{~ms}$ sampling time. 


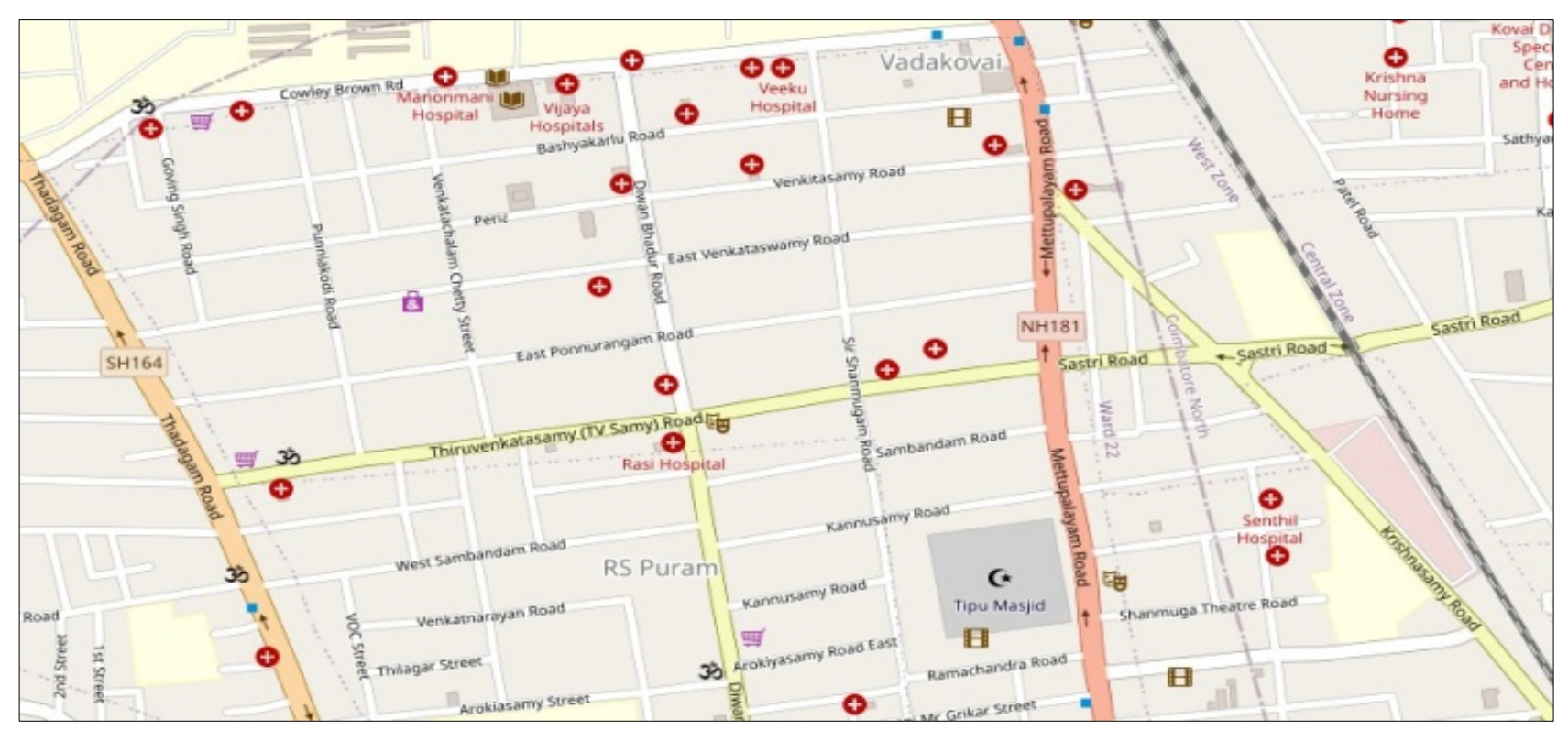

Fig. 5. Street map of Coimbatore city OSM file

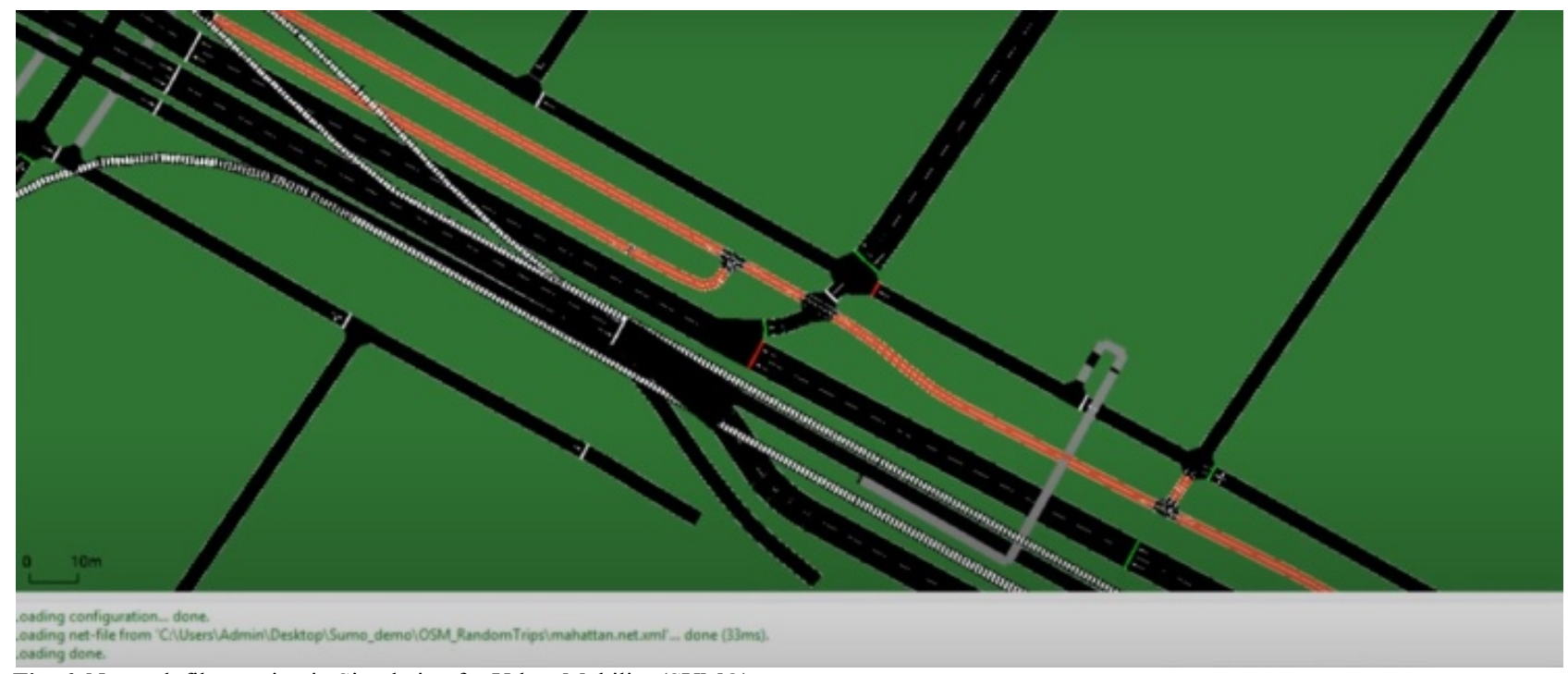

Fig. 6. Network file running in Simulation for Urban Mobility (SUMO)

Table 2. Scenario number according to vehicle density

\begin{tabular}{l|l}
\hline Scenario Number & Vehicle Density \\
\hline 1 & Less than 10 \\
2 & $10-30$ \\
3 & $30-50$ \\
4 & $50-70$ \\
5 & $70-90$ \\
6 & $90-120$ \\
7 & $120-130$ \\
8 & $130-140$ \\
9 & $140-160$ \\
10 & $160-180$ \\
11 & $180-200$ \\
\hline
\end{tabular}

\subsection{Performance Metrics}

The following six performance metrics are considered for evaluating the performance of the proposed method: Root Mean Square Error (RMSE), Prediction Accuracy, Beacon Reduction Rate, Broadcasting Interval, Beacon Broadcasting Rate and Beacon Receiving Rate [29].

Root Mean Square Error: RMSE is used for evaluation the level of error caused by the broadcasting algorithms. RMSE is the average distance between the predicted position and the actual position. Equation illustrates the formulation of RMSE, where $\mathrm{n}$ is the total number of terms for which the RMSE is to be calculated. $\mathrm{xi}$ is the observed value, and ${ }^{\wedge} \mathrm{xi}$ is the predicted value [22].

$\mathrm{RMSE}=\sqrt{\frac{\sum_{i=1}^{n}\left(\breve{x}_{i}-x_{i}\right)^{2}}{n}}$

Position Accuracy: The second metric is the position accuracy, which is the variation between the actual position of the broadcasting or neighbor vehicle and the predicted position using AEKF method. The position is predicted for $\mathrm{BV}$ before constructing the beacon and $\mathrm{AV}$ after receiving the beacon message holding BV's position. To achieve proper communication reliability, the position accuracy is very important. The efficiency of the broadcasting algorithm is determined by its prediction accuracy [23].

Beacon Reduction Rate: The Third metric is the Beacon Reduction Rate, which is the number of beacon messages granted for transmitting out of the total generated messages. 
It is important to note that not all the generated messages are granted for transmitting. An efficient broadcasting algorithm should achieve high Beacon Reduction Rate [24].

Broadcasting Interval: The fourth performance metric is the Broadcasting Interval (BI), which is the standard time period between the beacon messages, which received successfully at the neighbor end in a second [25].

Beacon Broadcasting Rate: Fifth performance metric is the Beacon Broadcasting Rate (BBR). BBR number of beacons broadcasted in a second. A good broadcasting algorithm should reduce the broadcasting of beacon messages in complicated situations. Increased number of beacons causes congestion, so that vehicles could not receive appropriate emergency messages. Finally affects the communication reliability [26].

Beacon Receiving Rate: Sixth performance metric is Beacon Receiving Rate, which is the ratio of beacons received successfully at the neighbor vehicle. Some beacons may loss due to the dynamic traffic situations. When reducing the Beacon Broadcasting Rate, the chance of Beacon receiving Rate will increase. This results in improving the communication reliability [27].

\subsection{Results and Discussion}

The performance of TCA-CBB is compared with Prediction Based Broadcasting Rate Control (PPBR) [18], Adaptive Beaconing Rate (ABR) [16] and Situation Adaptive Beaconing (SAB) [15]. PPBR decides whether to send the beacon message or not based on the prediction accuracy of maneuver or non-maneuvering models. ABR is the based on the EKF-Baseline prediction algorithm, which is used to predict, lost or omitted beacon messages. SAB depends on road traffic situation and the vehicle status in consideration with the currently available load. The evaluation is based on the real communication scenario created by NGSIM dataset [30]. The vehicle density is grouped into different scenarios as seen in Table 2. First performance metric, RMSE is plotted for proposed TCA-CBB method and existing broadcasting algorithms such as PPBR, ABR and SAB. As shown in figure 7, the proposed method appears to be low in RMSE error making. According to table 2, when scenario number is 1 (i.e vehicle density less than 10), TCA-CBB method produces only $7 \%$ of RMSE, whereas PPBR, ABR and SAB produces $12 \%, 15 \%$, and $20 \%$ RMSE respectively. Next, figure 8 .a shows the position predicted by four broadcasting schemes comparing with the actual $\mathrm{BV}$ position. It can be seen that the proposed TCA-CBB method accurately predicts the BV position. The position predicted by other three broadcasting algorithms such as PPBR, ABR and SAB varies more than 90 $\mathrm{cm}$. Likewise; the position is predicted at the Adjacent Vehicle (AV) segment is shown in figure 8.b. On seeing the graph in figure 8.b, it is clear that the proposed TCA-CBB method plots the AV position more accurately. Here, the position predicted by other three schemes varies more than $95 \mathrm{~cm}$. Based on BV and AV position prediction, the overall prediction accuracy is evaluated. Figure 9 shows the level of accuracy gained by each broadcasting algorithm. From this graph, it is seen that the proposed TCA-CBB method could gain more than $98.99 \%$ of prediction accuracy during scenario number 11. Other broadcasting algorithms such as PPBR, ABR and SAB bring accuracy about $90.31 \%, 89.6 \%$ and $85.23 \%$ respectively. Next performance metric is Beacon Reduction Rate.

An efficient broadcasting algorithm should reduce the number of beacons transmitted in order to maintain communication reliability [31]. Figure 10 shows the Beacon Reduction Rate estimated by the broadcasting algorithms. TCA-CBB produces $98 \%$ of Beacon Reduction Rate, whereas PPBR, ABR and $\mathrm{SAB}$ produce $80 \%, 85 \%$ and $70 \%$ of Broadcasting Reduction Rate respectively during scenario number 11. Beacon Broadcasting Interval is a major factor for efficient performance of the broadcasting algorithm [32]. Higher broadcasting interval reduces the number of beacon packets automatically, which results in network reliability [33]. Figure 11 shows the Broadcasting Interval (BI) achieved by the broadcasting algorithms. Broadcasting Interval gradually decreases from 30.45 seconds 18.26 seconds in TCA-CBB method. That means TCA-CBB takes 18.26 seconds to transmit a beacon packet after a successful delivery at the adjacent vehicle side during scenario number 11. Other broadcasting algorithms such as PPBR take 13.24 seconds, ABR take 10.23 seconds and $\mathrm{SAB}$ take 9.2 seconds to transmit a packet, not considering about the successful beacon delivery. Total number of beacons transmitted for an effective communication is mentioned as Beacon Broadcasting Rate (BBR). Figure 12 shows the graph of Beacon Broadcasting Rate (BBR) generated by each broadcasting algorithm. Totally 44 beacons where generated by SAB and 37 beacons where generated by ABR. PPBR generated 32 beacons in a complete transmission. The proposed TCA-CBB method generated 27 beacons at the 11th scenario. This evaluation shows that the proposed method gradually decreases the Beacon Broadcasting Rate. At the adjacent vehicle side, the beacons should receive successfully. However, the lost or omitted message is reconstructed at the adjacent vehicle side. Even though, the proposed method gives $100 \%$ of success rate at scenario 1 and gives $94.21 \%$ of success rate at scenario 11 . Whereas the existing methods brings averagely $76.54 \%$ of success of beacon delivery at scenario 11. The graph of Beacon Receiving Rate is shown figure 13. Form these simulation results, the proposed TCA-CBB method shows effective performance over other three broadcasting algorithms used for comparison. Thus, TCA-CBB is proved as a good quality-broadcasting algorithm for VANET applications.

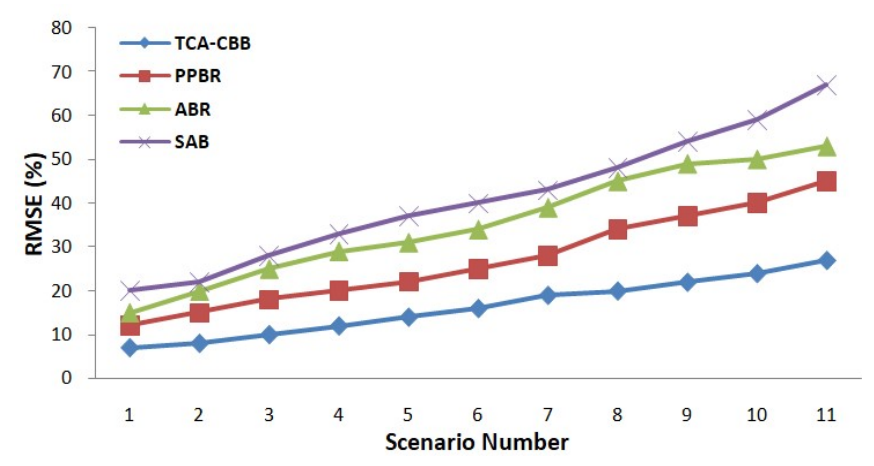


Fig. 7. RMSE (\%) Assessment

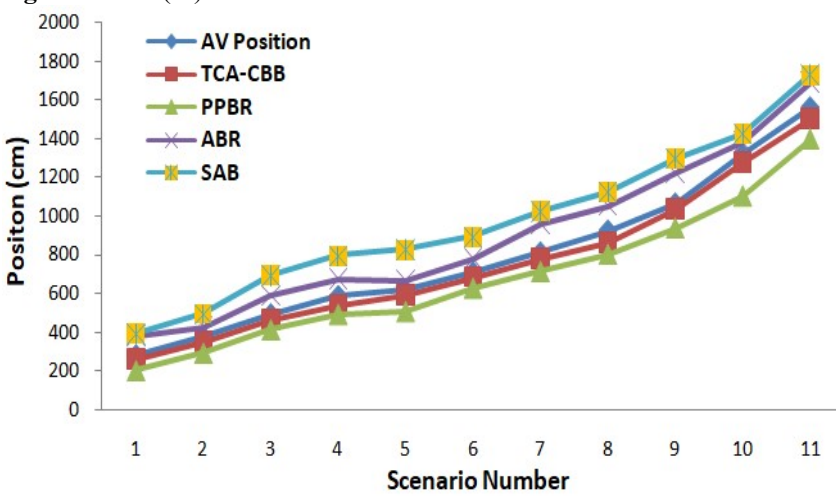

(a)

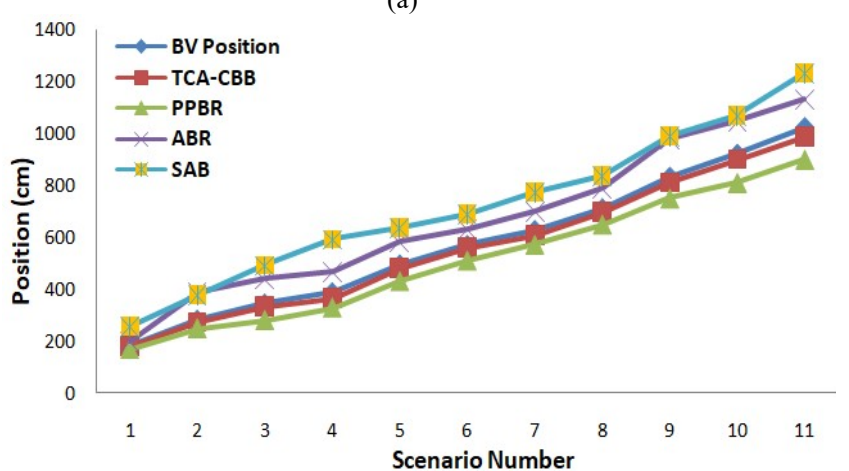

(b)

Fig. 8. (a) Position Prediction Accuracy (cm) in BV.

(b) Position Prediction Accuracy $(\mathrm{cm})$ in AV

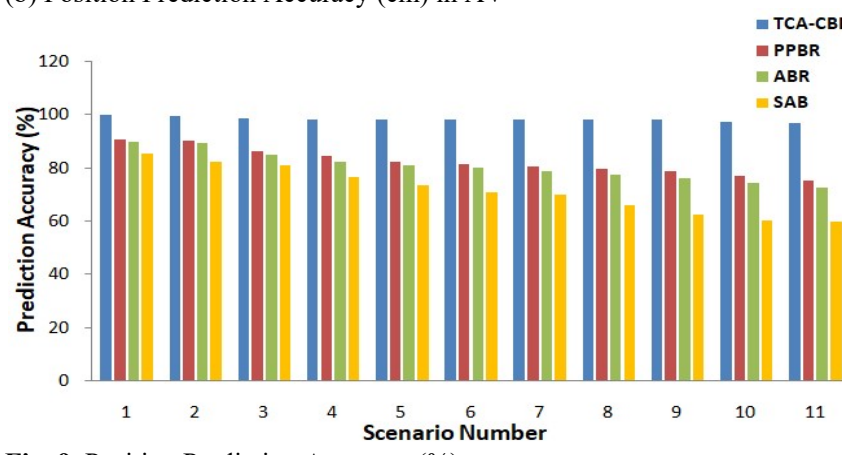

Fig. 9. Position Prediction Accuracy (\%)

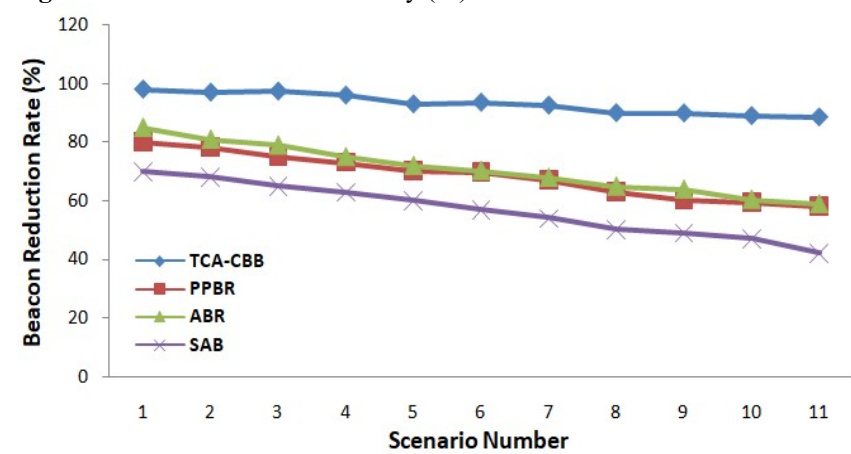

Fig. 10. Beacon Reduction Rate (\%)

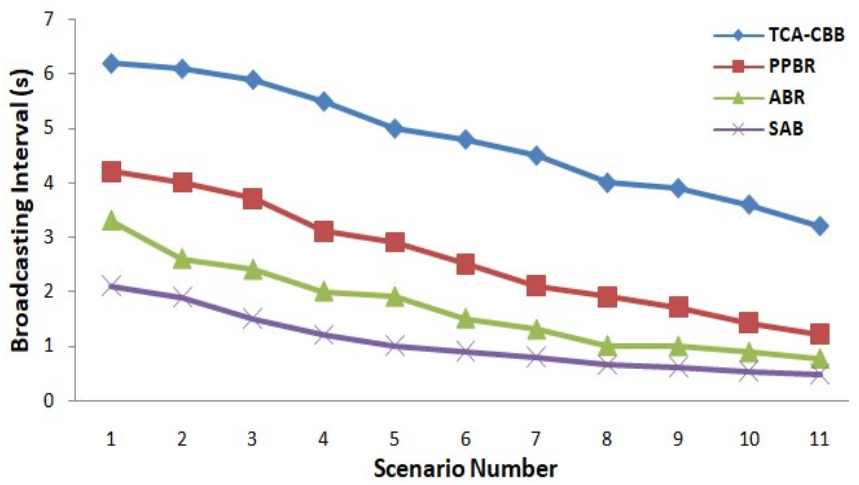

Fig. 11. Broadcasting Interval (s)

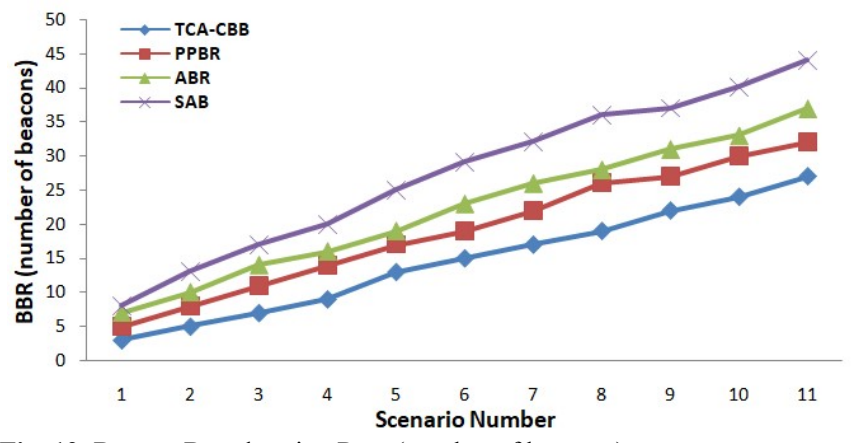

Fig. 12. Beacon Broadcasting Rate (number of beacons)

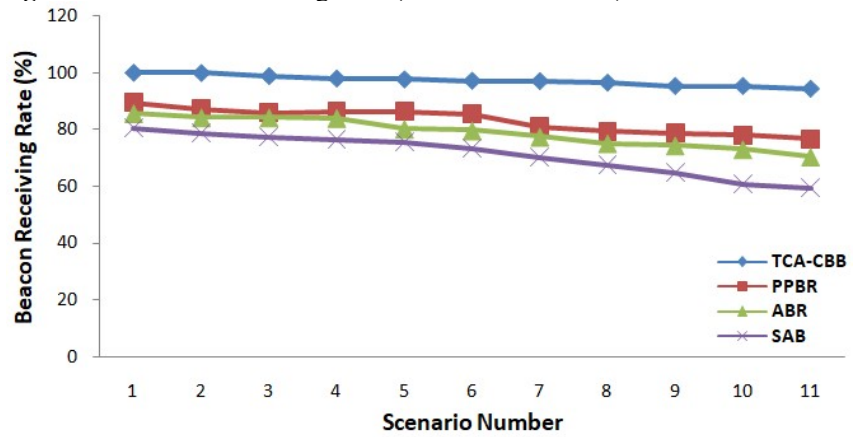

Fig. 13. Beacon Receiving Rate (\%)

\section{Conclusion}

In Vehicular Adhoc Network (VANET), each vehicle should exchange their mobility information in suitable rate to maintain better performance and communication reliability of the VANET application. Due to the dynamic nature of VANET, high broadcasting rate results in accessing mobility information and bandwidth and produces lack of communication among the vehicles. Since the traffic condition and the environmental behavior changes rapidly, fixed broadcasting rate for all sort of traffic situations causes unreliable network. There are chances of beacon losing. In this paper a broadcasting algorithm namely Traffic Condition Aware Customized Beacon Broadcasting Scheme (TCA$\mathrm{CBB}$ ) is proposed. TCA-CBB is introduced to analyze the traffic condition and adaptively fix or change the broadcasting rate in order to improve the network reliability and accuracy. Existing broadcasting schemes scarifies the information accuracy to bring effective broadcasting. In the view that, each and every individual vehicle mobility in VANET can be steady for a while, considering prediction process and driver behavior can enhance the network reliability and mobility information accuracy. The performance of the proposed method is compared with three existing broadcasting algorithms such as PPBR, ABR and SAB. SUMO traffic simulator, NS2 with MATLAB, does the simulation of the 
proposed method. The simulation results show that the proposed method TCA-CBB outperforms other three broadcasting algorithms such as PPBR, ABR and SAB.
This is an Open Access article distributed under the terms of the Creative Commons Attribution License.

\section{References}

1. S.E. Shladover and S.-K. Tan, "Analysis of Vehicle Positioning Accuracy Requirements for Communication-Based Cooperative Collision Warning”, Journal of Intelligent Transportation Systems Vol.10, No. 3, pp.131-140, 2006.

2. Kayhan Zrar Ghafoor, Jaime Lioret, et al. "Beaconing Approaches in Vehicular Ad Hoc Networks: A Survey", Wireless Personal Communications Vol.73, No.3, pp.885-912, 2013.

3. K. Golestan, F. Sattar, F. Karray, "Localization in vehicular ad hoc networks using data fusion and V2V communication", Computer Communications, Vol.71, pp.61.72, 2015.

4. K.A. Hafeez, Lian Zhao, Bobby Ma, Jon Mark, "Performance analysis and enhancement of the DSRC for VANET's safety applications", IEEE Transactions on Vehicular Technology, Vol.62, No.7, pp. 3069-3083, 2013.

5. H. Hartenstein and K.P. Laberteaux, "A tutorial survey on vehicular ad hoc networks", Communications Magazine, IEEE, Vol.46, No.6, pp.164-171, 2008.

6. Y. Hou, P. Edara and C. Sun, "Modeling mandatory lane changing using bayes classifier and decision trees", IEEE Transactions on Intelligent Transportation Systems, Vol.15 No.2, pp.647-655, 2014.

7. G. Karagiannis, et al., " Vehicular Networking: A Survey and Tutorial on Requirements, Architectures, Challenges, Standards and Solutions", Communications Surveys \& Tutorials, IEEE, Vol.13, N0.4, pp.584-616, 2011.

8. J. Huang and H.S. Tan, "Error analysis and performance evaluation of a future-trajectory-based cooperative collision warning system", IEEE Transactions on Intelligent Transportation Systems, Vol. 10, No.1, pp.175-180, 2009.

9. H. Hartenstein, VANET: "Vehicular Applications and InterNetworking Technologies", Wiley, Chichester, UK, 2010.

10. Y. Mo, et al., "A beacon transmission power control algorithm based on wireless channel load forecasting in VANETs", PLoS ONE, Vol.10, No.11, 2015.

11. Y. P. Fallah,C.-L. Huang, R. Sengupta, and H. Krishnan, "Analysis of information dissemination in vehicular ad-hoc networks with application to cooperative vehicle safety systems", IEEE Transactions on Vehicular Technology, Vol. 60, No. 1, pp. 233-247, 2011.

12. M. Torrent-Moreno, J. Mittag, P. Santi, and H. Hartenstein, "Vehicle-to-vehicle communication: fair transmit power control for safety-critical information", IEEE Transactions on Vehicular Technology, Vol. 58, No. 7, pp. 3684-3703, 2009.

13. K.P. Shih, Y.D. Chen and C.C. Chang, " A physical/virtual carriersense-based power control MAC protocol for collision avoidance in wireless ad hoc networks", IEEE Transactions on Parallel and Distributed Systems, Vol.22, No.2, pp.193-207, 2011.

14. Y. Park and H. Kim, "Application-level frequency control of periodic safety messages in the IEEEWAVE", IEEE Transactions on Vehicular Technology, Vol. 61, No. 4, pp. 1854-1862, 2012.

15. S. Rezaei, R. Sengupta and H. Krishnan, "Reducing the Communication Required By DSRC-Based Vehicle Safety Systems", IEEE Intelligent Transportation Systems Conference, 2007.

16. R. Schmidt and Tim Leinmüller, "Exploration of adaptive beaconing for efficient intervehicle safety communication", Network, IEEE, Vol.24, No.1, pp.14-19, 2010.

17. K. ZrarGhafoor, et al., "A fuzzy logic approach to beaconing for vehicular ad hoc networks, Telecommunication Systems", Vol. 52, No.1, pp.139-149, 2013.
18. S. Zemouri, S. Djahel and J. Murphy, "A short-term vehicular density prediction scheme for enhanced beaconing control", IEEE Global Communications Conference (GLOBECOM), 2015.

19. J. Liu and Q. Wang, "Position prediction based frequency control of beacons in vehicular ad hoc networks", International Journal of Distributed Sensor Networks, Volume 2015, Article ID 631415, pp. $10,2015$.

20. X. Yin, X. Ma and K.S. Trivedi, "MAC and application level performance evaluation of beacon message dissemination in DSRC safety communication", Performance Evaluation, Vol.71, pp.1-24, 2014.

21. W. Yan, "Toward automatic time-series forecasting using neural networks", IEEE Transactions on Neural Networks and Learning Systems, Vol. 23, No. 7, pp. 1028-1039, 2012.

22. G. P. Zhang and D. M. Kline, "Quarterly time-series forecasting with neural networks", IEEE Transactions on Neural Networks, Vol. 18, No. 6, pp. 1800-1814, 2007.

23. M.S. Frigau, "Cross-layer transmit power and beacon rate adaptation for VANETs", in Proceedings of the third ACM international symposium on Design and analysis of intelligent vehicular networks and applications, ACM: Barcelona, Spain. pp. 129-136, 2013.

24. 'K.A. Hafeez, et al., "Performance analysis and enhancement of the DSRC for VANET's safety applications", IEEE Transactions on Vehicular Technology, Vol. 62, No.7, pp.3069-3083. 2013.

25. S. Dietzel, et al., "A flexible, subjective logic-based framework for misbehavior detection in V2V networks", World of Wireless, Mobile and Multimedia Networks (WoWMoM), IEEE $15^{\text {th }}$ International Symposium on. 2014.

26. H. Wymeersch, J. Lien and M.Z. Win, "Cooperative localization in wireless networks", Proceedings of the IEEE. Vol.97, No.2, pp. 427$450,2009$.

27. T.L. Willke, P. Tientrakool and N.F. Maxemchuk, "A survey of inter-vehicle communication protocols and their applications, Communications Surveys \& Tutorials", IEEE. Vol.11, No.2, pp.320, 2009.

28. B.M.Williams and A. Guin, "Traffic management center use of incident detection algorithms: Findings of a nationwide survey", Intelligent Transportation Systems, IEEE Transactions, Vol.8, No.2, pp.351-358, 2007.

29. Sumithra, S., and R. Vadivel, "An overview of various trust models for vanet security establishment", In 2018 9th International Conference on Computing, Communication and Networking Technologies (ICCCNT), pp. 1-7. IEEE, 2018.

30. O.A. Wahab, H. Otrok and A. Mourad, "A cooperative watchdog model based on Dempster-Shafer for detecting misbehaving vehicles", Computer Communications, Vol.41, pp.43-54, 2014.

31. Sumithra, S., and R. Vadivel, "NB-FTBM model for entity trust evaluation in vehicular ad hoc network security", In International Conference on Ubiquitous Communications and Network Computing, pp. 173-187. Springer, Cham, 2019.

32. J. Tian, C. Lv, et al., "Connectivity Based Transmit Power Control in VANET", 8th International Wireless Communications and Mobile Computing Conference, pp.505-509, 2012.

33. V. Punzo, M.T. Borzacchiello and B. Ciuffo, "On the assessment of vehicle trajectory data accuracy and application to the Next Generation SIMulation (NGSIM) program data", Transportation Research Part C: Emerging Technologies Vol.19, No.6, pp.12431262, 2011. 